\title{
A clinicopathological correlation of the expression of the angiopoietin-Tie-2 receptor pathway in the brain of adults with Plasmodium falciparum malaria
}

Panote Prapansilp ${ }^{1,2,3^{*}}$, Isabelle Medana ${ }^{1}$, Nguyen Thi Hoan Mai ${ }^{4}$, Nicholas PJ Day ${ }^{1,2}$, Nguyen Hoan Phu ${ }^{4}$, Tsin W Yeo ${ }^{5}$, Tran Tinh Hien ${ }^{4}$, Nicholas J White ${ }^{1,2}$, Nicholas M Anstey ${ }^{4}$ and Gareth DH Turner ${ }^{1,2,3}$

\begin{abstract}
Background: Plasma angiopoietin (Ang)-2 is associated with disease severity and mortality in adults and children with falciparum malaria. However the mechanism of action of the angiopoietins in fatal malaria is unclear. This study aimed to determine whether the expression of Ang-1 and Ang-2 and their receptor Tie-2 in cerebral endothelial or parenchymal cells was specific to cerebral malaria (CM), correlated with coma or other severe clinical features, and whether plasma and CSF levels of these markers correlated with the clinical and neuropathological features of severe and fatal malaria in Vietnamese adults.

Methods: Immunohistochemistry was performed for Ang-1, Ang-2 and Tie-2 on post-mortem brain tissue from fatal malaria cases and controls. Quantitative ELISA for plasma and cerebrospinal fluid levels of Ang-1, Ang-2 and Tie-2 was done to compare fatal cases with surviving patients from the same study.

Results: Immunohistochemistry revealed significant differences in expression in endothelial and parenchymal cells compared to controls. However there was no significant difference in expression of these markers on endothelial cells, astroglial cells or neurons between CM and non-cerebral malaria cases. Immunostaining of Ang-1, Ang-2 and Tie-2 was also not associated with Plasmodium falciparum-infected erythrocyte sequestration in the brain. However Ang-1 and Ang-2 expression in neurons was significantly correlated with the incidence of microscopic haemorrhages. Plasma levels of Ang-2 and Ang-2/Ang-1 ratio were associated with the number of severe malaria complications and were significant and independent predictors of metabolic acidosis and fatal outcome.

Conclusions: The independent prognostic significance of Ang-2 and the Ang-2/Ang-1 ratio in severe malaria was confirmed, although immunohistochemistry in fatal cases did not reveal increased expression on brain endothelium in cerebral versus non-cerebral cases. Activation of the Ang-Tie-2 pathway in severe malaria is therefore related to acidosis, number of severity criteria and outcome, but is not a specific event in the brain during cerebral malaria.
\end{abstract}

Keywords: Malaria, Human, Plasmodium falciparum, Pathogenesis, Pathology, Cerebral, Angiopoietin, Tie-2 receptor, Immunohistochemistry, Biomarker, Blood brain barrier

\footnotetext{
* Correspondence: panote.prapansilp@gmail.com

${ }^{1}$ Nuffield Department of Medicine, Centre for Tropical Medicine, Oxford University, Oxford, UK

${ }^{2}$ Mahidol-Oxford Research Unit, Faculty of Tropical Medicine, Mahidol

University, 3rd Floor, 60th Anniversary Chalermprakiat Building, 420/6 Rajvithi

Road, Bangkok 10400, Thailand

Full list of author information is available at the end of the article
} 


\section{Background}

Malaria continues to be a major cause of mortality and morbidity in tropical countries [1]. Severe Plasmodium falciparum malaria, is characterized clinically using a series of criteria (WHO, 2000) [2], including complications such as hyperparasitaemia, metabolic acidosis, acute renal failure, severe anaemia and coma, presenting as cerebral malaria (CM). CM is a neurological syndrome comprising a potentially reversible diffuse encephalopathy, associated with retinopathy and variably with convulsions and localizing neurological signs, which are poor prognostic features [3].

The pathogenesis of CM is complex and multifactorial. A fundamental pathophysiological question in the genesis of coma in CM is how parasitized red blood cells (PRBC), remaining within the vascular space, influence parenchymal function to induce coma and death. Neurological dysfunction in severe malaria is significantly related to the sequestration of PRBC in the cerebral microvasculature, and resultant microvascular obstruction [4-7]. Both local and systemic factors may contribute to endothelial activation and blood brain barrier (BBB) dysfunction, causing secondary neuropathological processes such as neuronal and astroglial dysfunction or axonal injury [8].

The endothelium, which is the critical interface between PRBC and the brain parenchyma, is considered to be central to the pathogenesis of CM. During severe malaria infection, there is systemic activation of EC including the cerebral EC of the BBB $[9,10]$. Activated endothelium has increased permeability through the disruption of endothelial transmembrane proteins, and shows upregulation of a variety of surface adhesion molecules including intercellular cell adhesion molecule-1 (ICAM-1), vascular cell adhesion molecule-1 (VCAM-1), P- and E-selectin [9,11-14]. Moreover, activated ECs rapidly exocytose pre-synthesized and stored molecules from Weibel-Palade bodies (WPB) in response to changes in the vascular micro-environment. Although the main constituent of WPB is von-Willebrand factor (vWF) and its propeptide, other molecules including P-selectin, CD63, interleukin-8, endothelin-1, tissue plasminogen activator and angiopoietin (Ang)- 2 are also released. The release of WPB is the initial step in the transition from a resting endothelial phenotype to activated responsive endothelium [15-18].

The Ang-Tie-2 ligand-receptor system is a key regulator of the maintenance of functional integrity in both quiescent and activated endothelium [19]. In most contexts, Ang-1-mediated Tie-2 signalling maintains endothelium in a steady state but Ang-2, which functions as an antagonist ligand of Tie-2, can destabilize this and sensitize endothelial response to inflammatory and angiogenic cytokines such as tumour necrosis factor (TNF), interleukin-1 and vascular endothelial growth factor (VEGF) [13]. In order to maintain the quiescent phenotype of endothelium, ECs need constitutive Tie- 2 signalling mediated by
Ang-1 binding, which is mainly released from pericytes, fibroblast and smooth muscle cells and incorporated into extracellular matrix after secretion [20]. Tie-2 phosphorylation involves several downstream signalling pathways including activating phosphatidylinositol 3-kinase and protein kinase $B$ pathways, and inhibiting nuclear factor (NF)-кB [21]. These pathways protect cells from apoptosis and loss of intercellular junctional complexes and inhibit inflammatory responses. Exogenous stimuli such as physical damage, hypoxia or impaired endothelial nitric oxide production cause rapid exocytosis of stored Ang-2 in WPB from the ECs and competitively bind to Tie-2 receptor, which negatively interferes with constitutive Ang-1mediated Tie-2 signalling $[18,22,23]$. Consequently, Ang2-Tie-2 binding results in destabilization of endothelium and enhances EC responsiveness to other cytokines. Ang-2 can, therefore, act as an autocrine dynamic regulator of the rapid adaptive response of endothelium [13].

Recent in vivo studies have implicated the dysregulation of angiopoietins in the pathogenesis of severe and fatal malaria. In adults with cerebral and non-cerebral severe malaria, plasma Ang-2 has been associated with reduced endothelial nitric oxide bioavailability and mortality with Ang-2 a better predictor of death than venous lactate [24]. In children, increased plasma Ang-2 and the Ang-2/1 ratio, and reduced Ang-1 have been associated with disease severity and risk of death in CM [25-29]. Nevertheless, the mechanisms by which Ang-Tie-2 pathway contributes to $\mathrm{CM}$ and non-cerebral severe malaria (NCM), and fatal outcome, remain unclear.

The therapeutic use of angiopoietins has been investigated in experimental murine models of brain ischemia, where Ang-1 inhibits BBB breakdown, stimulates the recruitment of neuroblasts and promotes behavioural recovery [30]. The Ang-Tie-2 signalling pathway could potentially be a logical target for neuroprotective adjunctive therapy in CM. However their tissue specific expression and specificity in a target organ such as the brain has not been studied in human fatal malaria cases.

Therefore, in this study, the site and expression levels of the proteins Ang -1 and -2 and their receptor Tie-2 were examined in brain tissue of patients who died from malaria, to determine whether there were patterns of expression specific to cerebral malaria. Levels of the circulating forms of these proteins were examined in plasma and CSF of a subset of these patients in order to correlate circulating angiogenic protein levels and their expression patterns in the brain with the clinical manifestations of severe malaria.

\section{Methods}

\section{Ethical approvals}

This retrospective case-control research study used archival brain, plasma and CSF specimens of fatal and 
non-fatal malaria patients, who were studied in a large double-blinded trial of artemether versus quinine for the treatment of severe malaria in Vietnam (AQ trial) [31]. Relatives gave written informed consent for entering the study. The brain specimens of non-malaria cases (normal controls) were from a collection of routine autopsy cases at John Radcliffe Hospital, Oxford, UK, where specific written consent for retention of brain tissue for research purposes had been given. Protocols for tissue sampling, storage and use for research were approved by the Ethical and Scientific Committee of the Centre for Tropical Diseases in Ho Chi Min City, Vietnam, OXTREC 029-02, COREC (C01.002) and Human Tissue Authority license number 12217.

\section{Selection of cases}

Malaria and non-malaria cases

A total of 81 adults were included in this clinicopathological study (Figure 1). Of 81, 63 were severe malaria cases selected from the AQ trial [31], admitted at the centre for Tropical Diseases in Ho Chi Min City, Vietnam between 1991 and 1995. These were diagnosed with malaria using serial blood films and subsequent blood PCR. All were classified according to the WHO 1990 criteria for severe falciparum malaria [32]. Eighteen of the 81 were normal controls from routine autopsy at John Radcliffe Hospital (Oxford, UK). These normal controls had clinically confirmed multi-organ dysfunction and a variety of different

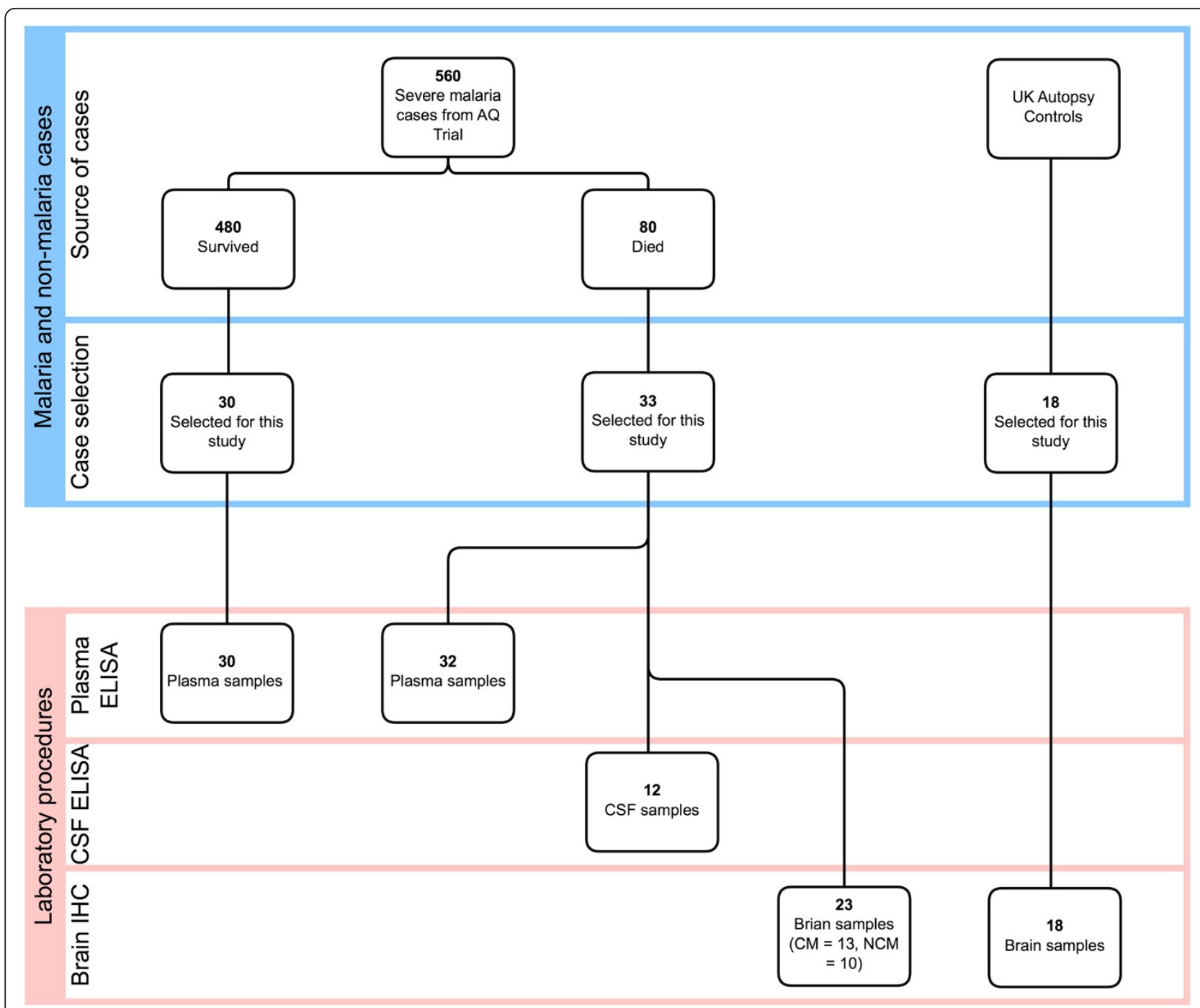

Figure 1 Schematic diagram to summarise case and sample selection. Samples were chosen through utility - cases where FFPE histological material was available, and as far as possible matching CSF and plasma samples to these cases, plus randomly selected other cases from the AQ study material to expand the numbers of fatal cases and survivors. 
causes of death, with normal appearances on subsequent neuropathological examination as previously detailed [33].

\section{Brain specimens}

In total, 41 cases were used. Twenty-three fatal malaria patients from the AQ study were examined, 13 of which were defined on $\mathrm{WHO}$ criteria as dying with $\mathrm{CM}$ and 10 were non-cerebral malaria (NCM). Brain sections from 18 normal controls from the UK were also used. For the malaria patients, brain specimens were collected at autopsy within 24 hours (median 7 hours), fixed in 10\% formalin, then embedded in paraffin and processed using standard methods as previously described [34]. Clinical details of fatal malaria and control cases are shown in Additional files 1 and 2, respectively.

\section{Plasma and CSF specimens}

Plasma samples from 62 out of 63 severe malaria cases were used for the quantitation of the angiogenic proteins by ELISA. CSF samples were available from only 12 severe malaria cases. Venous blood samples were taken immediately after admission into EDTA anticoagulant. Plasma samples were generated by centrifugation at $5,000 \mathrm{~g}$ for $3 \mathrm{~min}$, then aliquoted and stored at $-80^{\circ} \mathrm{C}$ until use. CSF was taken when the procedure was clinically indicated, aliquoted neat without any additives, spun and frozen at $-80^{\circ} \mathrm{C}$ until use [35].

\section{Clinical, biochemical and neuropathological data for correlations}

The cases used in this retrospective study had subsequent extensive clinical, biochemical and neuropathological investigations [31,33-38]. These existing data were correlated with immunostaining scores and plasma levels of Ang-1, Ang-2 and Tie-2 proteins generated from this study. These included assessment of the number of criteria of severe malarial disease, coma status, biochemical evidence of other end organs disease such as creatinine level for renal failure, liver function tests and lactate, peripheral blood counts for anaemia, white cell count and platelet levels, and neuropathological quantitation of sequestration, malarial haemorrhages and degree of oedema in individual cases. Data on CSF levels of the neurotransmitters quinolinic acid and kynurenine ratios [35] were also used.

\section{Detection and quantification of Ang-1, -2 and Tie-2 proteins in the brain \\ Immunohistochemistry}

Immunohistochemistry (IHC) was performed for three different markers of Ang-Tie-2 pathway including the Ang-1 protein, Ang-2 and Tie- 2 receptor. Staining was performed on $4 \mathrm{~mm}$ sections of formalin-fixed paraffin embedded brain blocks cut onto coated immunoslides (SupaFrost), from all malaria and control cases. The sections were dewaxed in xylene, rehydrated in graded alcohol series and then underwent microwave antigen retrieval in TrisEDTA $\mathrm{pH} 8$ for $10 \mathrm{~min}$. The primary antibodies used were: Ang-1 (mouse polyclonal, A0604, Sigma, UK, 1:20 dilution), Ang-2 (rabbit polyclonal, Ab65835-100, Abcam, UK, 1:50 dilution) and Tie-2 (rabbit polyclonal, SC-9026, Santa Cruz, USA, 1:100 dilution). Detection of bound antibody was visualized using the high-sensitivity Novolink $^{\mathrm{TM}}$ Polymer detection system (Leica Biosystems, UK). Negative controls comprised sections immunostained as above with omission of the primary antibody.

Three areas of the brain from each case were examined including cortex, diencephalon and brain stem, to determine any site specific variations in the patterns of immunoreactivity. With each brain area, the pattern and intensity of staining were examined in three different cellular components including vessels, neurons and astroglial cells.

\section{Quantitation of vascular immunostaining}

Vessels were considered positive for Ang-1, Ang-2 and Tie-2 when any parts forming the vessel including ECs, basement membrane, pericytes or smooth muscles were stained. The number of positive vessels in 150-300 fields in each slide at x200 magnification was used to calculate the number of positive vessels per centimetre squared. The continuous variable of number of positive vessels per $\mathrm{cm}^{2}$ was converted to a semiquantitative Vascular Expression Score (VasExp) as follows: Ang-1; low $=0-20$ vessels $/ \mathrm{cm}^{2}$, moderate $=20-40$ vessels $/ \mathrm{cm}^{2}$, high $\geq 40$ vessels $/ \mathrm{cm}^{2}$ : Ang2 ; low $=0-200$ vessels $/ \mathrm{cm}^{2}$, moderate $=200-400$ vessels $/$ $\mathrm{cm}^{2}$, high $\geq 400$ vessels $/ \mathrm{cm}^{2}$ : Tie-2; low $=0-10$ vessels $/ \mathrm{cm}^{2}$, moderate $=10-20$ vessels $/ \mathrm{cm}^{2}$, high $\geq 20$ vessels $/ \mathrm{cm}^{2}$. Both scores were used in histopathological and clinicopathological correlations where continuous or semiquantitative categorical measurements where most appropriate.

\section{Semiquantitation of neuronal and astroglial immunostaining}

Ang-1 and Ang-2 expression on neurons and astroglial cells was classified in a semi-quantitative scoring system of both the number of cells and the intensity of expression. Cell numbers were classified according to a percentage score as follows: score $0=$ percentage of stained cells $1 \%$ or less, score $1=1-10 \%$, score $2=10-50 \%$ score $3 \geq 50 \%$. The intensity score was defined as: score $0=$ no staining, score 1 = weak staining $(+/-)$, score $2=$ moderate staining $(+)$, score $3=$ strong staining $(++)$. The neuronal expression (NeuExp) of Ang-1 and Ang-2 was represented as the sum of the percentage score and intensity score (NeuScore) and also divided into two groups of "low" (low NeuExp, NeuScore $\leq 3$ ) and "high" neuronal expression (high NeuExp, NeuScore $\geq 4$ ). Astroglial expression (GliExp) of Ang-1 and Ang-2 was classified based on a similar system combining the percentage score with the 
intensity score into "low" (low GliExp, GliScore $\leq 3$ ) and "high" astroglial expression (high GliExp, GliScore $\geq 4$ ). However, due to much lower expression of Tie-2 on neurons and astroglia cells, it could not be quantitated using the same scoring system as Ang-1 and Ang-2. Rather than using both the number of cells and the intensity of staining, the semi-quantitative scoring system for Tie-2 expression on neurons and astroglial cells was based on the number of cells staining as follows: "low" neuronal or astroglial expression (low NeuExp or GliExp) = percentage of stained cells 1\% or less and "high" neuronal or astroglial expression (high NeuExp or GliExp) = percentage of stained cells $>1 \%$.

\section{Quantification of Ang-1, -2 and Tie-2 proteins in the plasma and CSF}

Plasma and CSF concentrations of Ang-1, Ang-2 and Tie-2 were measured by quantitative ELISA (R\&D Systems, UK), according to the manufacturer's instructions, each sample tested in duplicate. Concentrations were interpolated from 5-parameter-logistic (5-PL) standard curve fitting, using the manufacturer's recombinant human Ang-1, Ang-2 and Tie-2 proteins.

A total of 62 plasma samples and 12 CSF samples from patients of severe malaria were examined, all in duplicate. Twenty-two of the 62 plasma samples and 12 CSF samples were from fatal malaria cases included in the immunohistochemical analysis. The other 40 plasma samples were from severe malaria patients in the same AQ trial, selected randomly, including fatal cases $(\mathrm{n}=10 / 40)$ and survivors ( $n=30 / 40)$.

\section{Statistical methods}

Analyses were performed using Stata/SE 9.2 (StataCorp LP, Texas, USA), with a $P$ value less than 0.05 taken as being statistically significant. Continuous variables were tested for normal distribution with the KolmogorovSmirnov test, (this showed that the distribution of all continuous variables measured was not normal). Thus data were summarized with median and interquartile values, and non-parametric tests, including KruskalWallis, Mann-Whitney U test, and Wilcoxon matchedpaired signed-ranks test were used to compare groups where appropriate. Correlations between continuous variables were tested by Spearman's rank correlation.

For IHC expression score data: due to the correlated nature of data obtained from different areas of the brain within the same patient (three areas of the brain from one patient), intraclass correlation, which affected the standard errors of the estimates, were taken into account by using clustered robust standard errors (one patient as one cluster) with univariate logistic or ordered logistic regressions where appropriate.
The prognostic value of plasma angiopoietins, Tie-2 and other biomarkers was assessed by calculating the area under the receiver operating characteristic curve (AUROC). The AUROCs were compared nonparametrically by DeLong's method.

Multivariate analyses were conducted by either linear regression or logistic regression, depending on the nature of the dependent variable. Stepwise techniques were used manually in the light of clinical judgments where appropriate.

\section{Results}

Patterns of Ang-1, Ang-2 and Tie-2 immunostaining in the brain

Ang-1, Ang-2 and Tie-2 staining could be found in the cytoplasm and/or nucleus of a range of cells, including neurons, astroglial cells, ECs, pericytes, and perivascular smooth muscle cells, in the brain sections of malaria and non-malaria patients (Figure 2). There was considerable heterogeneity in the expression of Ang-1, Ang-2 and Tie-2 between patients and different brain areas of each patient. The expression of Ang-1 and Ang-2 was more commonly seen on larger neurons or astroglial cells than smaller neuron or astroglial cells. All three proteins could be seen staining vascular components including ECs, pericytes and perivascular smooth muscles, but Ang-1 expression was almost never seen on ECs. All three angiogenic markers were also observed in axonal fibres, intravascular serum and red blood cells either with or without parasites. However, Tie-2 staining on astroglial cells was exclusively found in malaria cases and not constitutive in controls.

\section{Comparative quantitation of Ang-1, Ang-2 and Tie-2 expression between different areas of the brain}

The expression of each marker was compared between the three different brain areas and in different cellular components. Detailed analysis of staining patterns of all cases (pooled malaria cases and non-malaria controls) and of malaria cases only is presented in Additional files 3 and 4, respectively.

Immunostaining of Ang-1, Ang-2 and Tie-2 differentiates malaria cases from controls but not cerebral malaria from non-cerebral malaria

Because the circulating levels of Ang-1, Ang-2 and Ang-2/ Ang-1 ratio have been reported to be clinically informative biomarkers for CM, we examined the expression patterns of proteins Ang-1, Ang-2 and their receptor Tie-2 in the brain tissue of patients dying of CM and compared them to NCM. No significant differences were found between the immunostaining patterns of Ang-1, Ang-2 and Tie-2 on all three cellular components including neurons, astroglial cells and vessels in CM cases compared to NCM (Figure 3). However, there were significant differences in 


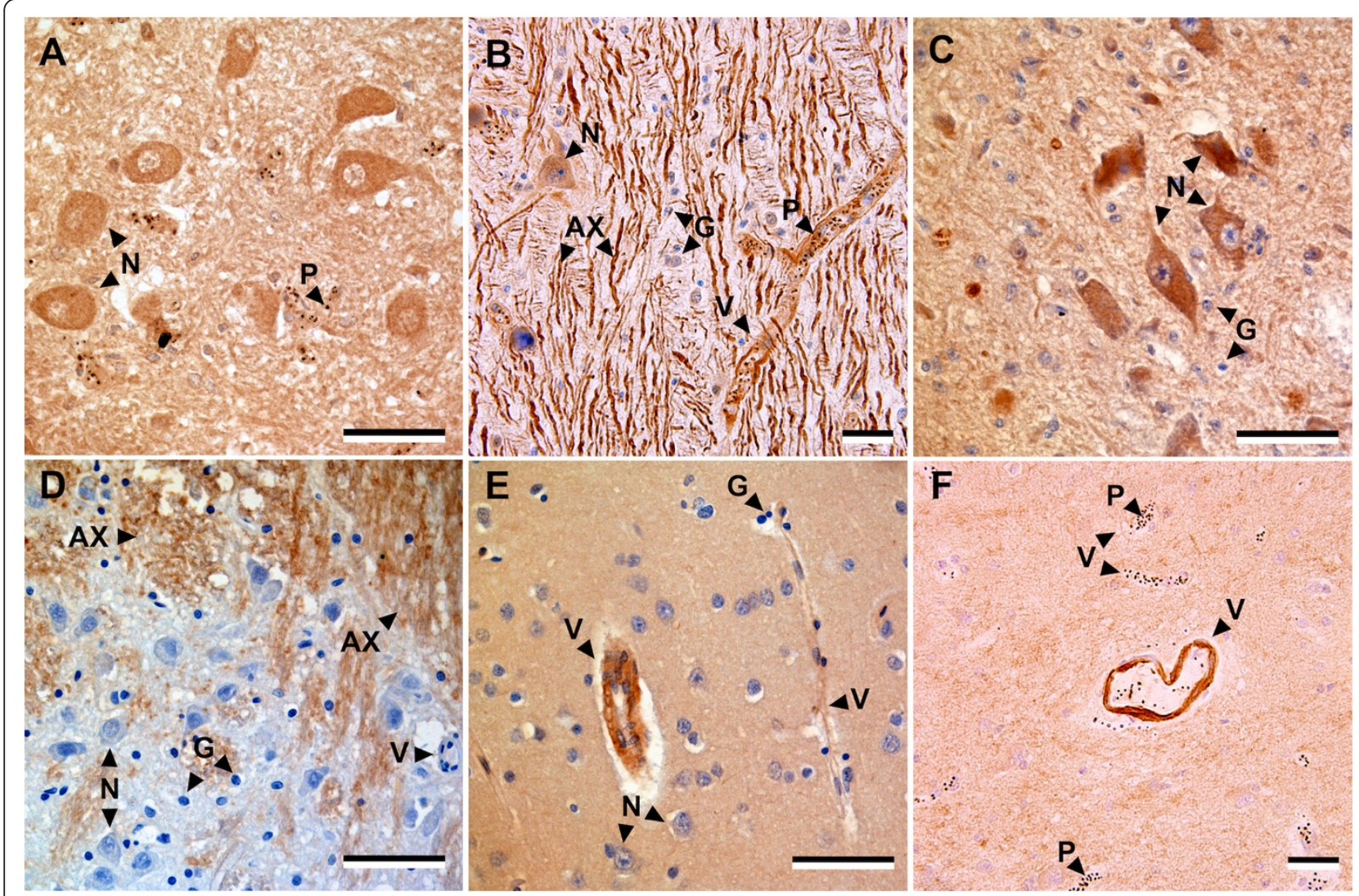

Figure 2 The immunostaining of Ang-1, Ang-2 and Tie-2 in brain sections from fatal malaria cases (A, B, C, F) and fatal non-malaria cases (D, E). There was heterogeneity in expression patterns of Ang-1, Ang-2 and Tie-2 between patients and within different brain regions in each patient. (A, D) Ang-1 was more commonly seen on larger neurons or astroglial cells, perivascular smooth muscles and pericytes, but not usually on endothelial cells (ECS). Strong staining was occasionally observed on neuropil and axonal bundles. (B, E) Strong Ang-2 labelling on axonal bundles, large neurons, astroglial cells, perivascular smooth muscles, pericytes, ECs and some parasitized red blood cells. (C, F) Strong Tie-2 staining of large and small neurons, astroglial cells, perivascular smooth muscles, pericytes and ECs. (F) A larger vessel showing Tie-2 staining whilst surrounding capillaries, showing significant parasitized erythrocyte sequestration, are completely negative. All sections were counterstained with haematoxylin. Scale bars $=60 \mu \mathrm{m}$. Abbreviations: $\mathrm{AX}=$ axons, $\mathrm{G}=$ astroglial cells, $\mathrm{N}=$ neurons, $\mathrm{P}=$ parasitized red blood cells, $\mathrm{V}=$ vessels.

the patterns of immunostaining of Ang-1, Ang-2 and Tie2 in malaria cases compared to non-malaria cases, especially in neurons and astroglial cells. Summaries of the staining patterns and odds ratios are shown in Figure 4.

\section{Ang-1}

The incidence of high Ang-1 expression on neurons and astroglial cells was greater in malaria cases $(73.91 \%$, $79.71 \%$, respectively), compared to non-malaria cases (35.19\%, 24.07\%, respectively) $(P<0.001)$. Ang-1 expression on vessels did not differ between malaria and nonmalaria cases.

\section{Ang-2}

The incidence of high Ang-2 expression on neurons was greater in brain sections of malaria cases $(65.22 \%)$, compared to non-malaria cases $(33.33 \%)(P=0.002)$. Ang-2 astroglial expression was lower in malaria compared to non-malaria $(P=0.078)$; which became statistically significant after adjusting for age and sex $(P=0.038)$.

\section{Tie-2}

Reduced expression of Tie-2 was observed in astroglial cells of malaria cases, all slides of malaria cases $(100 \%)$ did not express Tie- 2 on astroglial cells, compared to $77.78 \%$ of non-malaria cases $(P<0.001$, Fisher's exact). The vascular expression of Tie-2 was significantly higher in malaria cases (high VasExp 40.58\%), compared to non-malaria cases (low VasExp 11.1\%) $(P=0.049)$. There was a trend of increased incidence of Tie-2 neuronal expression in malaria compared to non-malaria $(P=0.059)$.

\section{Increased expression of Ang-1 and Ang-2 in neurons is associated with microscopic haemorrhages}

Microscopic cerebral haemorrhages are a common but non-specific neuropathological finding in CM [8]. Two main types of haemorrhage are seen including simple 


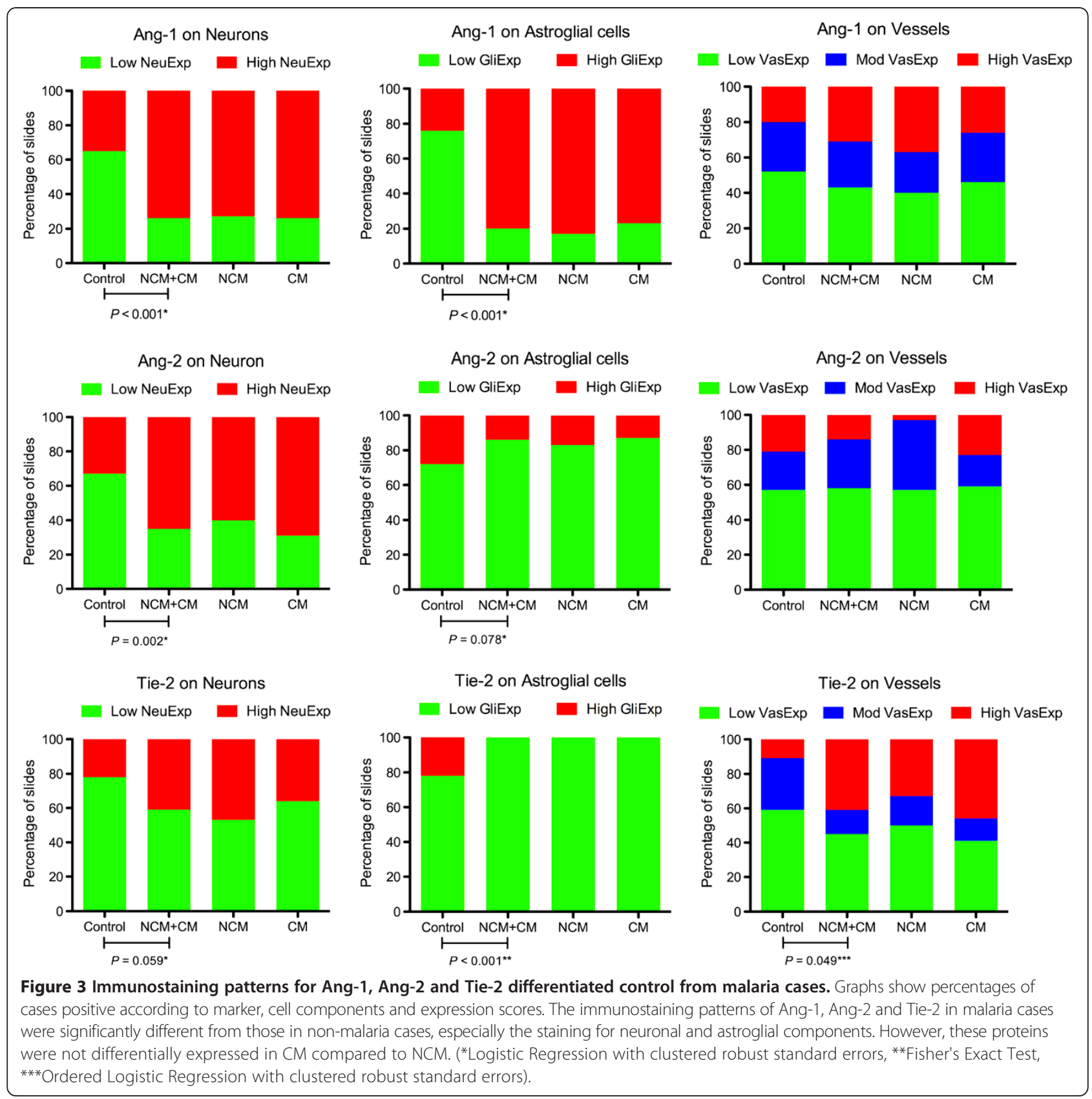

petechial haemorrhage or organized ring haemorrhages (Figure 5). Both types of haemorrhage are not exclusively specific to CM, but also present in NCM and other diseases such as bacterial infections and thrombotic phenomena such as bone marrow embolism or barotrauma. They represent focal damage to the microvasculature and hence the regulation of EC barrier function. Therefore, we examined the correlation between immunostaining patterns of Ang-1, Ang-2 and Tie2 and microscopic haemorrhage in the brain.

Within the brain sections of malaria cases in this study, $40.6 \%$ showed haemorrhages. Petechial haemorrhages
(88.9\%) were more common than ring haemorrhage (11.1\%). No significant difference in the incidence of haemorrhages was found between $\mathrm{CM}$ and NCM in this series. There was no correlation between the vascular or astroglial expression of Ang-1, Ang-2 or Tie-2 and the incidence or number of haemorrhages per $\mathrm{cm}^{2}$. However, high NeuExp of Ang-1 (OR = 7.11, 95\%CI = 1.50-33.64) was associated with the presence of microscopic haemorrhages $(P=0.013)$.

Taking all clinical groups (malaria and non-malaria) into account, high NeuExp of Ang-1 (OR $=13.30,95 \% \mathrm{CI}=$ 3.27-54.09) and Ang-2 ( OR $=5.5,95 \% \mathrm{CI}=1.77-17.01)$ 


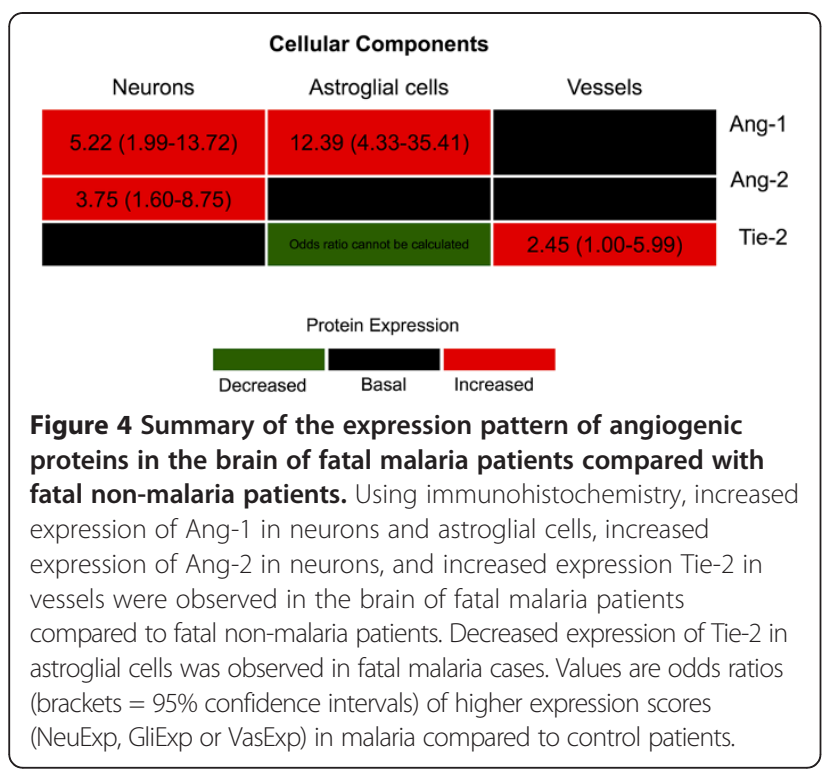

was significantly associated with the presence of haemorrhage $(P=0.003, P<0.001$, respectively).

\section{Immunostaining of Ang-1, Ang-2 and Tie-2 is not} associated with parasite sequestration in the brain

No correlation was found between the degree of PRBC sequestration in cerebral microvessels (measured as the percentage of vessels containing histologically observed PRBC) and the expression of any of the angiogenic proteins in either vessels, astroglial cells or neurones.

\section{CSF levels of circulating Ang-1, Ang-2 and Tie-2 are much lower than those in plasma}

Sixty-two plasma samples of malaria patients were included. Only a limited number of CSF samples were available from cases where IHC had been performed on the corresponding case $(\mathrm{n}=12)$. Although this did not provide enough power to determine the association of the CSF levels of the angiogenic markers with the clinical groups or parameters, the CSF levels of Ang-1, Ang-2 and Tie-2 had not been previously examined in malaria patients. Median and inter-quartile range of CSF and plasma levels of Ang-1, Ang-2 and Tie-2 are shown in Table 1. There were no correlations between baseline plasma concentrations of Ang-1, Ang-2 and Tie-2. The CSF concentrations of all three markers were significantly lower than the plasma concentration of themselves in the same cases (ranging from three to 800 times) ( $P=0.0005$, Wilcoxon matched-paired signed-ranks test). However this group was too small to determine any association between CSF levels and either plasma levels of the same marker, or clinical outcome.

\section{Plasma levels of Ang-2 and Ang-2/Ang-1 ratio are significant predictors of fatal outcome}

The level of Ang-2 and the ratio of Ang-2/Ang-1 (plasma samples collected at time of admission) were significantly higher in patients with fatal outcome than survivors $(P=0.002$ and $P=0.029$, respectively). Ang- 1 and Tie- 2 concentrations were not associated with fatal outcome (Table 1).

In order to assess the prognostic value of Ang-2 and Ang-2/Ang-1 ratio in assessing a fatal outcome in severe malaria patients, receiver operating characteristic (ROC) curves were plotted, and the areas under the ROC (AUROC) were compared. ROC curves of other laboratory measures previously shown to be important prognostic markers of death in severe malaria were also plotted and compared with those of Ang-2 and Ang-2/Ang-1 ratio. These included plasma creatinine, lactate and TNF. Ang-2

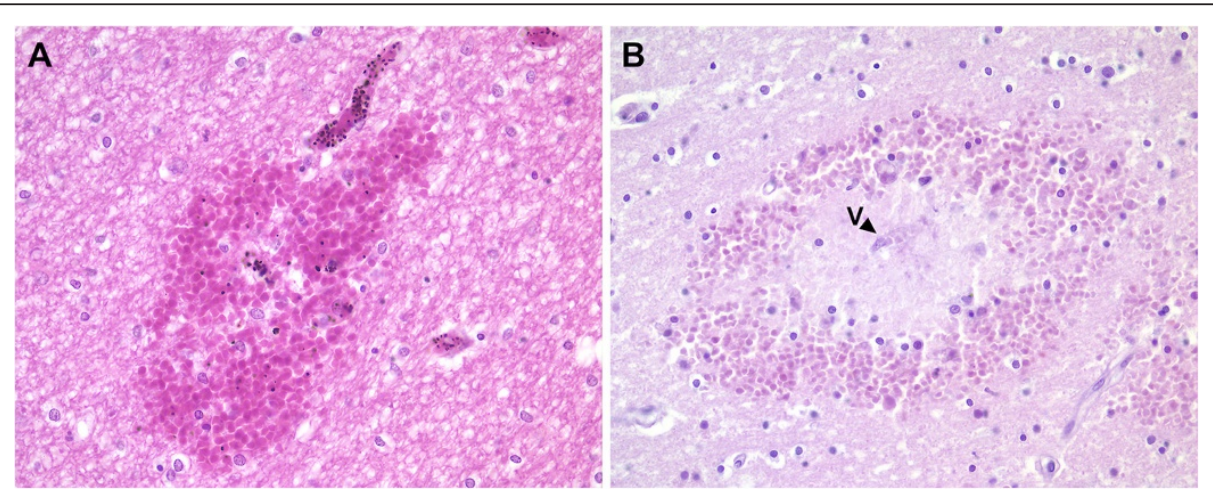

Figure 5 Malaria hemorrhages. Photomicrographs to demonstrate the histological characteristics of simple petechial haemorrhage (A) and a classical ring haemorrhage (B) in the brain tissues of a case of cerebral malaria. The simple petechial haemorrhage shows a feeding vessel containing sequestered parasitized erythrocytes and an area of leakage of infected and uninfected erythrocytes into the brain parenchyma. In contrast the ring haemorrhage shows a central vessel $(V)$, surrounded by a zone of clearing and astroglial response, with an outer zone of erythrocytes and leukocytes which have been extravasated from the ruptured vessel. Such haemorrhages may contain fibrin platelet thrombi in the vascular lumen (Haematoxylin and Eosin staining x400). 
Table 1 Correlation of circulating Ang-Tie-2 concentrations and clinical complications in malaria patients

\begin{tabular}{|c|c|c|c|c|c|}
\hline & & Ang-1 (pg/ml) & Ang-2 (pg/ml) & Tie-2 (pg/ml) & Ang-2/Ang1 Ratio \\
\hline $\operatorname{CSF}(n=12)$ & & $10.27(8.1-11.1)$ & 609 (388-1059) & $247.6(119-343)$ & - \\
\hline Plasma $(n=62)$ & & $543.15(401-837)$ & $21126(11590-33595)$ & $28232(22425-33418)$ & $39.25(23.14-62.56)$ \\
\hline \multirow[t]{3}{*}{ Fatal outcome } & No $(n=30)$ & $591.36(377.8-862.7)$ & 14883 (9369-24849) & $28232(17288-33106)$ & 30.55 (18.00-48.28) \\
\hline & Yes $(n=32)$ & $495.33(401.0-825.2)$ & 28215 (17040-42921) & 28213 (22684-34395) & 42.54 (30.57-70.07) \\
\hline & P-Value* & 0.95 & 0.002 & 0.68 & 0.029 \\
\hline \multirow[t]{3}{*}{ Hyper-parasitaemia } & No $(n=41)$ & $688.94(412-976)$ & $18706(11590-33565)$ & $26013(22684-34277)$ & 31.26 (18.39-53.22) \\
\hline & Yes $(n=21)$ & $495.33(377-615)$ & $23863(16110-34426)$ & 28709 (22425-32990) & $55.63(41.60-69.26)$ \\
\hline & P-Value* & 0.08 & 0.36 & 0.88 & 0.01 \\
\hline \multirow[t]{3}{*}{ Jaundice } & No $(n=19)$ & 459.77 (263-787) & 9692 (7064-12949) & 22907 (15334-29245) & $21.94(8.18-60.95)$ \\
\hline & Yes $(n=43)$ & 591.36 (401-963) & $25041(18288-41865)$ & $28824(22981-37659)$ & $41.60(30.17-63.16)$ \\
\hline & P-Value* & 0.13 & $<0.0001$ & 0.016 & 0.031 \\
\hline \multirow[t]{3}{*}{ Anaemia } & No $(n=23)$ & 495.33 (401-787) & 12848 (9369-24519) & 29245 (24960-33418) & $28.10(18.00-44.05)$ \\
\hline & Yes $(n=39)$ & 555.18 (377-988) & $26565(13657-41865)$ & $23800(19090-35373)$ & 41.70 (30.55-71.30) \\
\hline & P-Value* & 0.92 & 0.004 & 0.19 & 0.009 \\
\hline \multirow[t]{3}{*}{ Acute renal failure } & No $(n=23)$ & 495.33 (377-713) & 11615 (8376-18706) & 27908 (22684-32990) & $30.10(18.00-43.75)$ \\
\hline & Yes $(n=39)$ & 664.38 (401-988) & $26788(18288-43978)$ & 28709 (21318-37659) & 41.60 (30.17-69.26) \\
\hline & P-Value* & 0.06 & $<0.0001$ & 0.58 & 0.06 \\
\hline \multirow[t]{3}{*}{ Hypoglycaemia } & No $(n=44)$ & $567.21(401-812)$ & 18497 (9829-26217) & 28805 (22907-34825) & $31.60(18.78-48.28)$ \\
\hline & Yes $(n=18)$ & $519.24(377-963)$ & $32948(13657-45867)$ & 23241 (19853-32679) & 57.99 (37.34-85.16) \\
\hline & P-Value* & 0.98 & 0.007 & 0.29 & 0.019 \\
\hline \multirow[t]{3}{*}{ Shocked } & No $(n=38)$ & 173.50 (401-988) & $19932(11590-31282)$ & 28767 (22684-37659) & $33.94(18.78-56-24)$ \\
\hline & Yes $(n=24)$ & $459.77(389-591)$ & $24191(11841-34918)$ & 27699 (21817-31751) & 43.62 (29.77-76.06) \\
\hline & P-Value* & 0.053 & 0.36 & 0.48 & 0.13 \\
\hline \multirow[t]{3}{*}{ Hyperlactataemia } & No $(n=39)$ & $688.94(401-988)$ & $18706(10415-33565)$ & $27718(21171-37659)$ & 32.66 (22.77-59.84) \\
\hline & Yes $(n=23)$ & $495.33(377-713)$ & $24601(12444-35352)$ & $28786(23501-32990)$ & 43.48 (23.14-70.88) \\
\hline & P-Value* & 0.14 & 0.36 & 0.69 & 0.2 \\
\hline \multirow[t]{3}{*}{ Cerebral malaria } & No $(n=29)$ & $495.33(308-812)$ & $21540(13657-31021)$ & 24960 (19090-37818) & $41.60(29.66-62.56)$ \\
\hline & Yes $(n=33)$ & $579.28(436-850)$ & 18706 (9692-34426) & 28709 (22918-32990) & $37.55(20.65-63.61)$ \\
\hline & P-Value* & 0.41 & 0.75 & 0.49 & 0.59 \\
\hline \multirow[t]{3}{*}{ Pure cerebral malaria } & No $(n=54)$ & $519.24(377-837)$ & $24191(12848-34485)$ & $28633(22425-35373)$ & 41.35 (29.66-66.28) \\
\hline & Yes $(n=8)$ & $713.50(471-862)$ & 7237 (5178-11321) & 25407 (19415-29744) & $8.18(5.65-20.55)$ \\
\hline & P-Value & 0.31 & $<0.0001$ & 0.27 & 0.002 \\
\hline
\end{tabular}

Values are presented as median (IQR), *Mann-Whitney $\mathrm{U}$ test.

has an area under curve (AUROC) of 0.727 (95\%CI $=0.6$ $0.85)$, and Ang-2/Ang-1 ratio has an AUROC of 0.662 (0.522-0.802). The prognostic values of Ang-2 and Ang-2/ Ang-1 ratio were not significantly different from each other or those of plasma creatinine, lactate and TNF (Table 2). These results confirm the utility of Ang-2 and Ang-2/Ang1 ratio as predictive markers of fatal outcome in severe malaria as shown in previous studies [24,39].

A multivariable logistic regression model was constructed to assess whether these angiogenic proteins were independently associated with death, using fatal outcome as the dependent variable and the angiogenic proteins as well as creatinine, lactate and TNF as the independent variables. In this model, none of the markers were independently significantly associated with death. Then backward stepwise approach was used and showed that Ang-2 (OR = 1.000036 per $\mathrm{pg} / \mathrm{ml}, 95 \% \mathrm{CI}=1.000004-1.000068)$ and lactate $(\mathrm{OR}=$ 1.21 per $\mathrm{mmol} / \mathrm{L}, 95 \% \mathrm{CI}=1.032-1.435)$ were independently associated with fatal outcome. Ang-2 and lactate remain significant prognostic markers after adding into the model other clinical and biochemical parameters, including age, sex, presence of shock, presence of hypoglycaemia, admission Glasgow coma score (GCS), admission haematocrit and parasite count. 
Table 2 Comparison of prognostic value of plasma marker concentrations for fatal outcome

\begin{tabular}{llll}
\hline Marker & AUC $(\mathbf{9 5} \% \mathrm{Cl})$ & Compared to Ang-2 & Compared to Ang-2/Ang-1 Ratio \\
\hline Ang2 & $0.727(0.600-0.854)$ & & \\
Ang2/Ang1 Ratio & $0.662(0.522-0.802)$ & $0.82,0.36$ & $0.22,0.64$ \\
Creatinine & $0.708(0.575-0.840)$ & $0.09,0.77$ & $0.01,0.93$ \\
Lactate & $0.666(0.528-0.804)$ & $0.4,0.52$ & $0.57,0.75$ \\
TNF $(n=32)$ & $0.696(0.528-0.863)$ & 0,1 & \\
\hline
\end{tabular}

AUC $=$ area under ROC curve, Data are $x^{2}, P$-value unless otherwise indicated. All $X^{2}$ and $P$-value are calculated from pairwise nonparametric comparison of areas under ROC curve.

Plasma levels of Ang-2 and Ang-2/Ang-1 ratio are associated with multiple clinical complications of severe malaria

High plasma levels of Ang-2 were significantly associated with the presences of several complications of severe malaria including fatal outcome, jaundice, anaemia, acute renal failure and hypoglycaemia (Table 1). High Ang-2/ Ang-1 ratios were also associated with the same clinical complications as Ang-2, but with slightly lower magnitude. Hyperparasitaemia was associated with high Ang-2/ Ang-1 ratio and low Ang-1 but not independently associated with raised Ang-2. High plasma levels of Tie-2 were associated with the presence of jaundice but not the other complications.

\section{Plasma levels of Ang-2 is not a biomarker for CM but a prognostic marker for progressive multi-organ dysfunction in severe malaria}

The presence of cerebral malaria was not related to the plasma concentrations of Ang-1, Ang-2 and Tie-2; however, patients with "pure" cerebral malaria (cerebral malaria patients without the other complications of severe malaria according to WHO 2000 criteria) had significantly lower Ang-2 concentration and Ang-2/Ang-1 ratio than the other patients (Table 1).

Plasma Ang-2 concentrations were significantly associated with the number of severe complications of severe malaria occurring in a patient $(P<0.0001$, Kruskal-Wallis $)$ (Table 3 ). This indicated that Ang-2 could be a prognostic marker of progressive multiple organ dysfunction in severe malaria. Conversely, there were no correlations between Ang-1 or Tie-2 concentrations and the number of severity criteria.

\section{Correlation of clinical and laboratory parameters with plasma levels of Ang-1, Ang-2 and Tie-2 proteins}

Spearman's rank correlations were calculated between plasma concentrations of Ang-1, Ang-2 or Tie-2 and a number of clinical and biochemical markers in malaria patients at admission, including GCS, respiratory rate, haematocrit, white blood cell count, platelets, parasite count, liver function tests, creatinine, glucose, electrolytes, lactate, TNF, and arterial blood gas measures. The summary of correlations of some important markers of malaria severity was presented in Table 4.

Plasma Ang-1 concentration was positively correlated with the admission levels of platelets. This finding may reflect platelet production and release of circulating Ang-1 [40]. However Ang-2 and Ang-2/Ang-1 ratio were negatively correlated with platelet counts. There were positive correlations between body temperature and concentrations of Ang-1 and Tie-2, raising the possibility that these proteins might play a role in body temperature regulation in response to the disease. Plasma Ang-2 was correlated with several indicators of disease activity/severity, including increased white cell count, increased liver enzymes (SGOT and SGPT), decreased renal function (elevated BUN, creatinine and potassium) and metabolic acidosis (low $\mathrm{pH}$, high $\mathrm{pCO}_{2}$ ). Moreover, plasma Ang-2 concentration was strongly positively correlated with CSF concentrations of quinolinic acid and picolinic acid, which have been previously shown to be strongly associated with acute renal failure in this group [38]. There were no correlations between admission parasite count and the plasma levels of Ang-1 Ang-2 or Tie-2, but admission parasite count was significantly correlated with the ratio of Ang-2/Ang-1.

Interestingly lactate and pyruvate levels, that could also contribute to metabolic acidosis, were not correlated with Ang-1, Ang-2 and Tie-2 levels. This finding suggested that Ang-2 was correlated with metabolic acidosis in a manner independent of lactate levels (Spearman's rho $=0.09, P=0.47$ ).

\section{Plasma levels of Ang-2 and lactate were independently} associated with metabolic acidosis

As described above, plasma Ang-2 levels were closely correlated with several established prognostic indices of malaria disease severity and several factors indicating disease activity. As the various clinical manifestations and laboratory parameters of severe malaria are interlinked, a backward stepwise multivariable linear regression model was constructed to determine the factors contributing to plasma Ang-2 level, with plasma level of Ang-2 as the dependent variable and haematocrit, parasite count, platelets, white cell counts, creatinine, SGOT, and lactate as possible explanatory factors. All of these 


\begin{tabular}{|c|c|c|c|}
\hline $\begin{array}{l}\text { No. of severe } \\
\text { criteria }\end{array}$ & $N$ & Ang-2 (pg/ml) & Ang-2/Ang1 \\
\hline 1 & $16(25.8 \%)$ & $9730(7237-15240)$ & $20.55(8.18-55.63)$ \\
\hline 2 & $28(45.2 \%)$ & 22616 (14883-34889) & $38.53(28.74-61.15)$ \\
\hline 3 & $8(12.9 \%)$ & $23532(15977-28215)$ & $51.66(17.19-67.77)$ \\
\hline 4 & $9(14.5 \%)$ & 34485 (31282-43978) & 41.60 (37.34-70.88) \\
\hline 5 & $1(1.6 \%)$ & 58192 & 94 \\
\hline Kruskal-Wallis & & $P<0.0001$ & $P=0.10$ \\
\hline
\end{tabular}

parameters independently and significantly associated with the level of Ang-2 in this model and together they are accounted for $67 \%\left(R^{2}=0.677\right)$ of the variance in Ang-2 (Model A, Table 5).

There was no correlation between lactate and Ang-2 in the univariate analysis, and that the multivariable logistic regression model predicting death as an outcome showed that Ang-2 and lactate were independent factors contributing to death; however, in this linear regression model (Model A, Table 5), lactate was a significant independent contributing factor to Ang-2. This suggested that there were other confounding factors related to the levels of Ang-2 and lactate. This could be metabolic acidosis, which is closely related as a complication of severe malaria to renal failure and hyperlactatemia, as reported elsewhere [38]. Admission arterial blood $\mathrm{pH}$, representing one measure of metabolic acidosis, was added into the model. Adding $\mathrm{pH}$ removed the independent significance of lactate, creatinine, and platelets, so these were removed from the model; only haematocrit, parasite count, white cell count and SGOT remained significant factors associated with levels of Ang-2 (Model B, Table 5). This supported the hypothesis that Ang-2 was not directly correlated with lactate but both of them (together with renal dysfunction) were correlated with one common manifestation of severe disease - metabolic acidosis.

\section{Conclusions}

Plasma concentrations of Ang-2 and the Ang-2/Ang-1 ratio were confirmed as independent predictors of death in Vietnamese adults with severe falciparum malaria, as seen in all other adequately powered severe malaria series studied to date. These include series of adult severe malaria from Indonesia [24], adult CM from India [25], and paediatric CM series from Uganda [26,27] and Malawi $[28,29]$. Patterns of immunostaining of Ang-1, Ang-2 and Tie-2 in malaria cases were altered in fatal severe malaria compared to fatal non-malaria cases, especially in neurons and astroglial cells. However, there were no specific patterns of Ang-1, Ang-2 and Tie-2 expression, either of neuronal, astroglial or vascular, that differentiated $\mathrm{CM}$ from non-cerebral severe malaria deaths. Activation of the Ang-Tie-2 pathway in severe malaria was related to acidosis, the number of severity criteria and outcome, but was not a specific event in the brain during $\mathrm{CM}$.

Previous pathological studies of CM have demonstrated the presence of EC activation in the cerebral microvasculature [9], which occurs as part of a wider systemic endothelial activation in severe malaria, and which is not specific to cases that develop coma [10]. The pathophysiology of endothelial activation in CM has been hypothesized to occur in response to both systemic factors (such as the release of cytokines or a soluble malaria toxin) and local vasoactive substances such as VEGF or WPB products such as Ang-2. The expression of mediators, which could influence endothelial reactivity in the brain in $\mathrm{CM}$ has been previously examined, including inducible nitric oxide synthase [41], the hypoxic inducible protein HIF-1a and activation of the VEGF signalling pathway [42]. Therefore this methodology was extended to examine the expression of the angiopoietins and their receptor Tie-2 in the brain in CM and NCM cases, to determine the extent to which they were specific to $\mathrm{CM}$ and whether this pathway showed a direct link to the clinical features of coma before death.

The lack of significant differences in the patterns or extent of staining of any of these constituents between $\mathrm{CM}$ and NCM cases is consistent with recent data using systemic measures of activation of the Ang-2-Tie2 pathway, where elevated plasma levels of Ang-2 were no higher in CM than in NCM [24]. Taken together, these results do not support elevated Ang-2 as a predictive biomarker for cerebral malaria, as previously proposed $[25,26]$. Indeed in the current study, circulating Ang-2 was elevated less in those with CM as the only manifestation than in those with other severe malaria criteria.

The low levels of Ang-2 expression in brain ECs in fatal $\mathrm{CM}$ and NCM, despite elevated plasma concentrations of Ang-2, was unexpected. It is possible that exocytosis of cerebral endothelial WPBs at the time of death decreased immunoreactivity, due to prolonged EC activation, as stored Ang-2 is rapidly released from WPBs on activation [18]. Alternatively, raised Ang-2 levels in serum may reflect release from the extra-cerebral systemic circulation in both CM and NCM.

As well as its independent association with fatal outcome, Ang-2 was associated with number of organ complications, metabolic acidosis, and acute renal failure, as previously shown [24]. In contrast, the other major WPB product increased in severe malaria, vWF, is not associated with either lactate/metabolic acidosis or fatal outcome in severe malaria $[39,43]$. Taken together, these findings do still suggest a specific pathogenic role for Ang- 2 in the pathways leading to death in severe malaria. Ang-2 competitively binds to Tie- 2 receptors blocking the homeostatic 
Table 4 Summary of correlations between plasma angiogenic markers and laboratory parameters

\begin{tabular}{|c|c|c|c|c|c|}
\hline & & Ang-1 & Ang-2 & Tie-2 & Ang-2/Ang1 Ratio \\
\hline \multirow[t]{2}{*}{ Body temperature } & rho, P-value* & $0.2788,0.029$ & ns & $0.441,<0.0001$ & \\
\hline & $\mathbf{n}$ & 61 & & 62 & \\
\hline \multirow[t]{2}{*}{ Haematocrit } & rho, P-value* & ns & $-0.368,0.003$ & ns & ns \\
\hline & $\mathbf{n}$ & & 62 & & \\
\hline \multirow[t]{2}{*}{ Parasite count } & rho, P-value* & ns & ns & ns & $0.3,0.018$ \\
\hline & $\mathbf{n}$ & & & & 61 \\
\hline \multirow[t]{2}{*}{ Platelets } & rho, P-value* & $0.305,0.018$ & $-0.294,0.022$ & ns & $-0.442,<0.0001$ \\
\hline & $\mathrm{n}$ & & 60 & & 59 \\
\hline \multirow[t]{2}{*}{ White blood cells } & rho, P-value* & ns & $0.471,<0.0001$ & ns & ns \\
\hline & $\mathrm{n}$ & & 59 & & \\
\hline \multirow[t]{2}{*}{ BUN } & rho, P-value* & ns & $0.733,<0.0001$ & ns & $0.554,0.002$ \\
\hline & $\mathbf{n}$ & & 27 & & 27 \\
\hline \multirow[t]{2}{*}{ Creatinine } & rho, P-value* & ns & $0.619,<0.0001$ & ns & $0.293,0.02$ \\
\hline & $\mathrm{n}$ & & 62 & & 61 \\
\hline \multirow[t]{2}{*}{ Potassium (K) } & rho, P-value* & ns & $0.311,0.027$ & ns & ns \\
\hline & $\mathbf{n}$ & & 50 & & \\
\hline \multirow[t]{2}{*}{ SGOT } & rho, P-value* & $0.317,0.014$ & $0.415,0.001$ & ns & ns \\
\hline & $\mathbf{n}$ & 59 & 60 & & \\
\hline \multirow[t]{2}{*}{ SGPT } & rho, P-value* & ns & $0.283,0.028$ & ns & ns \\
\hline & $\mathbf{n}$ & & 50 & & \\
\hline \multirow[t]{2}{*}{ TNF } & rho, P-value* & $0.416,0.017$ & ns & ns & ns \\
\hline & $\mathbf{n}$ & 32 & & & \\
\hline \multirow[t]{2}{*}{ Lactate } & rho, P-value* & ns & ns & ns & ns \\
\hline & $\mathbf{n}$ & & & & \\
\hline \multirow[t]{2}{*}{ Pyruvate } & rho, P-value* & ns & ns & ns & ns \\
\hline & $\mathrm{n}$ & & & & \\
\hline \multirow[t]{2}{*}{$\mathrm{PH}$} & rho, P-value* & ns & $-0.42,0.004$ & ns & ns \\
\hline & $\mathbf{n}$ & & 44 & & \\
\hline \multirow[t]{2}{*}{$\mathrm{pCO} 2$} & rho, P-value* & ns & $-0.4832,<0.0001$ & ns & $-0.438,0.003$ \\
\hline & $\mathrm{n}$ & & 44 & & 43 \\
\hline \multirow[t]{2}{*}{ CSF Kynurenine } & rho, P-value* & ns & ns & ns & ns \\
\hline & $\mathrm{n}$ & & & & \\
\hline \multirow[t]{2}{*}{ CSF Quinolinic Acid } & rho, P-value* & ns & $0.711,<0.0001$ & ns & $0.625,<0.0001$ \\
\hline & $\mathbf{n}$ & & 31 & & 30 \\
\hline \multirow[t]{2}{*}{ CSF Picolinic Acid } & rho, P-value* & ns & $0.753,<0.0001$ & ns & $0.708,<0.0001$ \\
\hline & $\mathbf{n}$ & & 31 & & \\
\hline
\end{tabular}

*Spearman's rank correlation. No adjustments for multiple comparison have been made. All parameters were measured from plasma samples (except indicated as CSF) at time of admission. ns = not significant.

effects of Ang-1, increasing endothelial activation and sensitising ECs to further activation and injury [19]. Ang-2 related amplification of endothelial activation, injury and microvascular sequestration may thus play a key role in impairment of microvascular perfusion in severe malaria.

Potential limitations to interpretation of immunohistochemical staining patterns include post mortem artefact, which may alter staining due to diffusion or degradation of proteins, and necessitated the use of control cases. Each death in an autopsy series gives a 'snapshot' effect of pathology in an individual case with varying treatment, time to death and clinical complications of disease, which differ between cases. Inferring a single unifying pattern of pathology with temporal sequence from a group of such cases 
Table 5 Summary of multivariate regression models

\begin{tabular}{|c|c|c|c|c|c|c|}
\hline Model & Dependent variable & Regression type & Independent variables & Coefficients $(95 \% \mathrm{Cl})$ & Standardized Coefficients & $P$ \\
\hline \multirow[t]{8}{*}{$A$} & Plasma Ang-2 & Linear regression & Haematocrit & $-506.65(-933$ to -80$)$ & -0.2294042 & 0.021 \\
\hline & & & Parasite count & 0.0116 (0.004 to 0.019$)$ & 0.2893166 & 0.003 \\
\hline & & $R^{2}=0.677$ & Platelet & -0.200 (0.333 to -0.067$)$ & -0.27261 & 0.004 \\
\hline & & & White cell & $1.260(0.746$ to 1.774$)$ & 0.4790173 & $<0.0001$ \\
\hline & & & Creatinine & 1965 (20.2 to 3911) & 0.2087163 & 0.048 \\
\hline & & & SGOT & 44.60 (19.61-69.58) & 0.3070279 & 0.001 \\
\hline & & & Lactate & $-1539(-2416$ to -661.7$)$ & -0.3230233 & 0.001 \\
\hline & & & Constant & 23369 (3808 to 42930) & - & 0.02 \\
\hline \multirow[t]{5}{*}{ B } & Plasma Ang-2 & Linear regression & Haematocrit & $-591.39(-1111$ to -71$)$ & -0.275 & 0.027 \\
\hline & & & Parasite count & 0.0111 (0.002 to 0.020$)$ & 0.280 & 0.017 \\
\hline & & $R^{2}=0.623$ & White cell & 1.44 (0.832 to 2.057) & 0.352 & $<0.0001$ \\
\hline & & & SGOT & 71.96 (23.07 to 120.85$)$ & 0.530 & 0.005 \\
\hline & & & Constant & $9200(-12672$ to 31072$)$ & - & 0.399 \\
\hline
\end{tabular}

may therefore be difficult. Severe malaria in Vietnamese adults is a multi-organ process with high prevalence of renal, liver, respiratory and metabolic dysfunction as well as anaemia and cerebral malaria. Hence several factors may affect the expression of a particular marker as judged by immunohistochemistry. Despite this, it was reassuring that the data on plasma levels of Ang-1, Ang-2 and Tie-2 were in keeping with previously published series on these mediators in severe and fatal malaria [24-29,39]. This lends weight to the findings that despite the significant increases in Ang-2 and Ang2/Ang-1 ratio in severe malaria, there was no evidence that this was a process that was specifically upregulated in the brain in $\mathrm{CM}$ versus NCM cases.

The analysis of the prognostic value of Ang-2 in severe malaria confirmed, as found in previous studies, that this marker is predictive of fatal outcome, at a level superior to previously studied prognostic biomarkers such as serum lactate. However most studies use a single (admission) value of serum Ang-2 and have not studied the kinetics during severe disease. Lactate levels fall rapidly after treatment but in contrast remain high or rise in patients with fatal outcome despite treatment $[44,45]$. Data on the kinetic responses of Ang-2 and Ang-2/Ang-1 ratio following treatment would be valuable in interpreting the specificity of Ang-2 increases in severe malaria.

The effects of Ang-1, Ang-2 and Tie-2 signalling on neurons and glial cells have not been as well studied as endothelium. It was notable that expression of Ang-1, Ang-2 and Tie-2 differed in neurons and glial cells in fatal malaria cases compared to controls. Recent studies indicate that Tie-2 signal transduction might have neuroprotective and mitogenic effects on neuronal cells. Ang-1-Tie-2 signalling promotes neural outgrowth from dorsal root ganglion cells [46], supports neuronal differentiation in neural progenitor cells [47], protects against neuronal apoptosis [48] and the effects of oxygen and glucose starvation [49], both of which may occur in the brain during the coma of CM. For these reasons, the increased neuronal Ang-1 expression in association with microvascular haemorrhages may reflect an adaptive, neuroprotective response to cerebral disease, where haemorrhages reflect focal damage to BBB function which are associated with perivascular edema formation [50]. In a murine model of angiogenesis during experimental autoimmune encephalomyelitis, temporal changes in neuronal and glial expression of Ang-1 and vascular expression of Tie-2 in the spinal cord was seen in concert with BBB permeability changes [51]. Ang-1 effects on neurons are not exclusively limited to Tie-2-receptor dependence, as Ang-1 can induce neurite outgrowth via the $\beta 1$-integrin receptor on neurons [52]. Other studies have also found a positive effect of Ang- 2 on neurogenesis and migration of neuroblasts [53]. It is therefore possible that increased expression of both Ang-1 and Ang-2 on neuronal cells are neuroprotective responses in severe malaria whether or not this involves coma.

Despite the finding that Ang-2 is associated with poor outcome, the immunohistochemical staining and ELISA data provided no evidence for direct involvement of this pathway in the genesis of coma by increases in CM versus NCM patients. The strong correlation between plasma Ang-2 levels and Ang2/Ang1 ratio with metabolic acidosis and increasing number of clinical severity criteria suggests an association with widespread endothelial activation in severe disease, even if this does not have a specific effect on BBB function in the genesis of coma. However the Ang-2-Tie-2 pathway could be studied using in vitro models of $\mathrm{BBB}$ function in the presence of sequestered, malaria-infected erythrocytes to investigate this further. Given the association of plasma Ang-2 with acute renal 
failure in this and other series, and the relationship with metabolic acidosis in the multivariate analysis, further studies should examine the role of the Ang-Tie-2 pathway in regulating blood flow and microvascular pathology in the kidney in adult severe malaria patients.

\section{Additional files}

Additional file 1: Clinical details of malaria cases used in the immunohistochemical study.

Additional file 2: Clinical details of control cases in the immunohistochemical study.

Additional file 3: Angiogenic markers expression in different cellular component across brain areas (pooled malaria cases and non-malaria controls).

Additional file 4: Angiogenic markers expression in different cellular component across brain areas (only malaria cases).

\section{Abbreviations}

CM: Cerebral Malaria; NCM: Non-Cerebral Malaria; BBB: Blood-Brain Barrier; Ang-1: Angiopoietin-1; Ang-2: Angiopoietin-2; vWF: von Willebrand Factor; EC: Endothelial Cell; WPB: Weibel-Palade Body; GCS: Glasgow Coma Scale; ELISA: Enzyme-Linked Immunosorbent Assay; WHO: World Health Organization; CSF: Cerebrospinal Fluid; TNF: Tumor Necrosis Factor; SGOT: Serum Glutamic Oxaloacetic Transaminase; SGPT: Serum Glutamic Pyruvic Transaminase; BUN: Blood Urea Nitrogen.

\section{Competing interests}

The authors declare that they have no competing interests.

\section{Authors' contributions}

PP carried the laboratory experiments, prepared and analysed the data obtained, drafted and finalized the manuscript for submission with contributions from authors. IM helped supervise and perform the laboratory experiments, analyse results and reviewed the manuscript. NTHM, NPJD, NHP, TTH, NJW, GDHT collected samples and data for the study. NJW, NPJD, TWY, NMA, GDHT conceived of the study, helped analyse results, wrote and reviewed the manuscript. All authors have read and approved the final manuscript.

\section{Acknowledgements}

We would like to thank the staff of the malaria ward, Hospital for Tropical Diseases in Ho Chi Minh City for their help with the autopsy trial. This study was funded with support from the Wellcome Trust (grant code 089275/Z/09/Z), The John Fell fund of Oxford University, and the Thrasher Research Foundation, USA.

\section{Author details}

${ }^{1}$ Nuffield Department of Medicine, Centre for Tropical Medicine, Oxford University, Oxford, UK. ${ }^{2}$ Mahidol-Oxford Research Unit, Faculty of Tropical Medicine, Mahidol University, 3rd Floor, 60th Anniversary Chalermprakiat Building, 420/6 Rajvithi Road, Bangkok 10400, Thailand. ${ }^{3}$ Department of Tropical Pathology, Faculty of Tropical Medicine, Mahidol University, Bangkok, Thailand. ${ }^{4}$ Hospital for Tropical Diseases, Ho Chi Minh City, Vietnam. ${ }^{5} \mathrm{Global}$ Health Division, Menzies School of Health Research and Charles Darwin University, Darwin, Australia.

Received: 25 October 2012 Accepted: 14 January 2013

Published: 5 February 2013

\section{References}

1. World Health Organization: World malaria report 2011. Geneva: World Health Organization; 2012.

2. World Health Organization: Severe falciparum malaria. Tran $R$ Soc Trop Med Hyg 2000, 94(Suppl 1):S1-S90.

3. Idro R, Otieno G, White S, Kahindi A, Fegan G, Ogutu B, Mithwani S, Maitland K, Neville BGR, Newton CRJC: Decorticate, decerebrate and opisthotonic posturing and seizures in Kenyan children with cerebral malaria. Malar J 2005, 4:57.

4. Pongponratn E, Riganti M, Punpoowong B, Aikawa M: Microvascular sequestration of parasitized erythrocytes in human falciparum malaria: a pathological study. AmJTrop Med Hyg 1991, 44:168-175.

5. Pongponratn E, Turner GDH, Day NPJ, Phu N, Simpson JA, Stepniewska K, Mai NTH, Viriyavejakul P, Looareesuwan S, Hien TT: An Ultrastructural study of the brain in fatal plasmodium falciparum malaria. AmJTrop Med Hyg 2003, 69:345-359.

6. Silamut K, Phu NH, Whitty C, Turner GDH, Louwrier K, Mai NTH, Simpson JA, Hien T, White NJ: A quantitative analysis of the microvascular sequestration of malaria parasites in the human brain. Am J Pathol 1999 155:395-410.

7. Dorovini-Zis K, Schmidt K, Huynh H, Fu WJ, Whitten RO, Milner DA, Kamiza SB, Molyneux ME, Taylor TE: The neuropathology of fatal cerebral malaria in Malawian children. Am J Pathol 2011, 178:2146-2158.

8. Medana IM, Turner GDH: Human cerebral malaria and the blood-brain barrier. Int J Parasitol 2006, 36:555-568.

9. Turner GDH, Morrison H, Jones M, Davis TM, Looareesuwan S, Buley ID, Gatter KC, Newbold Cl, Pukritayakamee S, Nagachinta B: An immunohistochemical study of the pathology of fatal malaria. Evidence for widespread endothelial activation and a potential role for intercellular adhesion molecule-1 in cerebral sequestration. Am J Pathol 1994, 145:1057-1069.

10. Turner GDH, Ly VC, Nguyen TH, Tran TH, Nguyen HP, Bethell DB, Wyllie S, Louwrier K, Fox SB, Gatter KC, Day NP, Tran TH, White NJ, Berendt AR: Systemic endothelial activation occurs in both mild and severe malaria. Correlating dermal microvascular endothelial cell phenotype and soluble cell adhesion molecules with disease severity. Am J Pathol 1998, 152:1477-1487.

11. Nag S, Manias J, Stewart DJ: Pathology and new players in the pathogenesis of brain edema. Acta Neuropathol 2009, 118:197-217.

12. Dejana $\mathrm{E}$, Tournier-Lasserve $\mathrm{E}$, Weinstein BM: The control of vascular integrity by endothelial cell junctions: molecular basis and pathological implications. Dev Cell 2009, 16:209-221.

13. Fiedler $\mathrm{U}$, Augustin $\mathrm{H}$ : Angiopoietins: a link between angiogenesis and inflammation. Trends Immunol 2006, 27:552-558.

14. Hawkins BT, Davis TP: The blood-brain barrier/neurovascular unit in health and disease. Pharmacol Rev 2005, 57:173-185.

15. Pfaff D, Fiedler U, Augustin HG: Emerging roles of the angiopoietin-Tie and the ephrin-Eph systems as regulators of cell trafficking. J Leuk Biol 2006, 80:719-726.

16. Pober JS, Sessa WC: Evolving functions of endothelial cells in inflammation. Nat Rev Immunol 2007, 7:803-815.

17. Rondaij M, Bierings R, Kragt A, van Mourik JA, Voorberg J: Dynamics and plasticity of weibel-palade bodies in endothelial cells. Arterioscler Thromb Vasc Biol 2006, 26:1002-1007.

18. Fiedler U, Scharpfenecker M, Koidl S, Hegen A, Grunow V, Schmidt JM, Kriz W, Thurston G, Hellmut G, Augustin HG: The Tie-2 ligand angiopoietin-2 is stored in and rapidly released upon stimulation from endothelial cell weibel-palade bodies. Blood 2004, 103:4150-4156.

19. Fiedler U, Reiss Y, Scharpfenecker M, Grunow V, Koidl S, Thurston G, Gale NW, Witzenrath M, Rosseau S, Suttorp N, Sobke A, Herrmann M, Preissner $K T$, Vajkoczy P, Augustin HG: Angiopoietin-2 sensitizes endothelial cells to TNF-alpha and has a crucial role in the induction of inflammation. Nat Med 2006, 12:235-239.

20. $\mathrm{Xu}$ Y: Angiopoietin-1, unlike angiopoietin-2, is incorporated into the extracellular matrix via its linker peptide region. J Biol Chem 2001, 276:34990-34998.

21. Makinde T, Agrawal DK: Intra and extravascular transmembrane signalling of angiopoietin-1-Tie2 receptor in health and disease. J Cell Mol Med 2008, 12:810-828.

22. Huang YQ, Li JJ, Hu L, Lee M, Karpatkin S: Thrombin induces increased expression and secretion of angiopoietin-2 from human umbilical vein endothelial cells. Blood 2002, 99:1646-1650.

23. Oh H, Takagi H, Suzuma K, Otani A, Matsumura M, Honda Y: Hypoxia and vascular endothelial growth factor selectively up-regulate angiopoietin-2 in bovine microvascular endothelial cells. J Biol Chem 1999, 274:15732-15739

24. Yeo TW, Lampah DA, Gitawati R, Tjitra E, Kenangalem E, Piera K, Price RN, Duffull SB, Celermajer DS, Anstey NM: Angiopoietin-2 is associated with 
decreased endothelial nitric oxide and poor clinical outcome in severe falciparum malaria. Proc Natl Acad Sci USA 2008, 105:17097-17102.

25. Jain V, Lucchi NW, Wilson NO, Blackstock AJ, Nagpal AC, Joel PK, Singh MP, Udhayakumar V, Stiles JK, Singh N: Plasma levels of angiopoietin-1 and -2 predict cerebral malaria outcome in central India. Malar J 2011, 10:383.

26. Conroy AL, Lafferty El, Lovegrove FE, Krudsood S, Tangpukdee N, Liles WC, Kain KC: Whole blood angiopoietin-1 and -2 levels discriminate cerebral and severe (non-cerebral) malaria from uncomplicated malaria. Malar J 2009, 8:295.

27. Lovegrove FE, Tangpukdee N, Opoka RO, Lafferty El, Rajwans N, Hawkes M, Krudsood S, Looareesuwan S, John CC, Liles WC, Kain KC: Serum angiopoietin-1 and -2 levels discriminate cerebral malaria from uncomplicated malaria and predict clinical outcome in African children. PLoS One 2009, 4:e4912.

28. Conroy AL, Phiri H, Hawkes M, Glover S, Mallewa M, Seydel KB, Taylor TE, Molyneux ME, Kain KC: Endothelium-based biomarkers are associated with cerebral malaria in Malawian children: a retrospective case-control study. PLoS One 2010, 5:e15291.

29. Conroy AL, Glover SJ, Hawkes M, Erdman LK, Seydel KB, Taylor TE, Molyneux $M E$, Kain KC: Angiopoietin-2 levels are associated with retinopathy and predict mortality in Malawian children with cerebral malaria: a retrospective case-control study. Crit Care Med 2012, 40:952-959.

30. Ohab JJ, Fleming S, Blesch A, Carmichael ST: A neurovascular niche for neurogenesis after stroke. J Neurosci 2006, 26:13007-13016.

31. Hien TT, Day NP, Phu NH, Mai NTH, Chau TT, White NJ: A controlled trial of Artemether or quinine in Vietnamese adults with severe falciparum malaria. NEJM 1996, 335:76-83.

32. World Health Organization: Severe and complicated malaria. Trans $R$ Soc Trop Med Hyg 1990, 84:1-65.

33. Medana IM, Mai NT, Day NP, Hien TT, Bethell D, Phu NH, Farrar J, White NJ, Turner GD: Cellular stress and injury responses in the brains of adult Vietnamese patients with fatal plasmodium falciparum malaria. Neuropathol Appl Neurobiol 2001, 27:421-433.

34. Medana IM, Day NPJ, Hien TT, Mai NTH, Bethell DB, Phu NH, Farrar J, Esiri MM, White NJ, Turner GDH: Axonal injury in cerebral malaria. Am J Pathol 2002, 160:655-666

35. Medana IM, Hien TT, Day NPJ, Phu NH, Mai NTH, Chuong LV, Chau TTH, Taylor A, Salahifar H, Stocker R, Smythe G, Turner GD, Farrar J, White NJ, Hunt NH: The clinical significance of cerebrospinal fluid levels of kynurenine pathway metabolites and lactate in severe malaria. J Infect Dis 2002, 185:650-656.

36. Ponsford MJ, Medana IM, Prapansilp P, Hien TT, Lee SJ, Dondorp AM, Esiri MM, Day NPJ, White NJ, Turner GDH: Sequestration and microvascular congestion are associated with coma in human cerebral malaria. J Infect Dis 2012, 205:663-671.

37. Day NPJ, Hien TT, Schollaardt T, Loc PP, Chuong LV, Chau TTH, Mai NTH, Phu NH, Sinh DX, White NJ, Ho M: The prognostic and pathophysiologic role of pro- and antiinflammatory cytokines in severe malaria. $J$ Infect Dis 1999, 180:1288-1297.

38. Day NPJ, Phu NH, Mai NTH, Chau TTH, Loc PP, Chuong LV, Sinh DX, Holloway P, Hien T, White NJ: The pathophysiologic and prognostic significance of acidosis in severe adult malaria. Crit Care Med 2000, 28:1833-1840.

39. Erdman LK, Dhabangi A, Musoke C, Conroy AL, Hawkes M, Higgins S, Rajwans N, Wolofsky KT, Streiner DL, Liles WC, Cserti-Gazdewich CM, Kain KC: Combinations of host biomarkers predict mortality among Ugandan children with severe malaria: a retrospective case-control study. PLOS One 2011, 6:e17440.

40. Li JJ, Huang YQ, Basch R, Karpatkin S: Thrombin induces the release of angiopoietin-1 from platelets. J Thromb Haemost 2001, 85:204-206.

41. Maneerat $Y$, Viriyavejakul $P$, Punpoowong $B$, Jones $M$, Wilairatana $P$, Pongponratn E, Turner GDH, Udomsangpetch R: Inducible nitric oxide synthase expression is increased in the brain in fatal cerebral malaria. Histopath 2000, 37:269-277.

42. Medana IM, Day NPJ, Roberts R, Sachanonta N, Turley H, Pongponratn E, Hien TT, White NJ, Turner GDH: Induction of the vascular endothelial growth factor pathway in the brain of adults with fatal falciparum malaria is a non-specific response to severe disease. Histopath 2010, 57:282-294

43. Phiri HT, Bridges DJ, Glover SJ, van Mourik JA, de Laat B, Mbaya B, Taylor TE, Seydel KB, Molyneux ME, Faragher EB, Craig AG, Bunn JE: Elevated plasma von willebrand factor and propeptide levels in Malawian children with malaria. PLoS One 2011, 6:e25626.

44. Krishna S, Waller DW, ter Kuile F, Kwiatkowski D, Crawley J, Craddock CF, Nosten F, Chapman D, Brewster D, Holloway PA, White NJ: Lactic acidosis and hypoglycaemia in children with severe malaria: pathophysiological and prognostic significance. Trans R Soc Trop Med Hyg 1994, 88:67-73.

45. Agbenyega T, Angus BJ, Bedu-Addo G, Baffoe-Bonnie B, Guyton T, Stacpoole PW, Krishna S: Glucose and lactate kinetics in children with severe malaria. J Clin Endocrinol Metab 2000, 85:1569-1576.

46. Kosacka J, Figiel M, Engele JR, Hilbig H, Majewski M, Spanel-Borowski K: Angiopoietin-1 promotes neurite outgrowth from dorsal root ganglion cells positive for Tie-2 receptor. Cell Tissue Res 2005, 320:11-19.

47. Bai Y, Cui M, Meng Z, Shen L, He Q, Zhang X, Chen F, Xiao J: Ectopic expression of angiopoietin-1 promotes neuronal differentiation in neural progenitor cells through the Akt pathway. Biochem Biophys Res Commun 2009, 378:296-301.

48. Valable S: Angiopoietin-1-induced phosphatidyl-inositol 3-kinase activation prevents neuronal apoptosis. FASEB J 2003, 17:443-445.

49. Bai $Y$, Meng Z, Cui M, Zhang X, Chen F, Xiao J, Shen L, Zhang Y: An Ang1Tie2-PI3K axis in neural progenitor cells initiates survival responses against oxygen and glucose deprivation. Neuroscience 2009, 160:371-381.

50. Medana IM, Day NPJ, Sachanonta N, Mai NTH, Dondorp AM, Pongponratn E, Hien TT, White NJ, Turner GDH: Coma in fatal adult human malaria is not caused by cerebral oedema. Malar J 2011, 10:267.

51. Macmillan CJ, Starkey RJ, Easton AS: Angiogenesis is regulated by angiopoietins during experimental autoimmune encephalomyelitis and is indirectly related to vascular permeability. J Neuropathol Exp Neurol 2011, 70:1107-1123.

52. Chen X, Fu W, Tung CE, Ward NL: Angiopoietin-1 induces neurite outgrowth of PC12 cells in a Tie2-independent, beta1-integrindependent manner. Neurosci Res 2009, 64:348-354.

53. Liu XS, Chopp M, Zhang RL, Buller B, Lu M, Zhang ZG: Angiopoietin 2 mediates the differentiation and migration of neural progenitor cells in the subventricular zone after stroke. J Biol Chem 2009, 284:22680-22689.

doi:10.1186/1475-2875-12-50

Cite this article as: Prapansilp et al:: A clinicopathological correlation of the expression of the angiopoietin-Tie- 2 receptor pathway in the brain of adults with Plasmodium falciparum malaria. Malaria Journal 2013 12:50.

\section{Submit your next manuscript to BioMed Central and take full advantage of:}

- Convenient online submission

- Thorough peer review

- No space constraints or color figure charges

- Immediate publication on acceptance

- Inclusion in PubMed, CAS, Scopus and Google Scholar

- Research which is freely available for redistribution 